\title{
Mamíferos del departamento del Tolima: distribución y estado de conservación
}

\section{Mammals of the Tolima department: distribution and state of conservation}

\author{
Leidy Viviana García-Herrera; Leidy Azucena Ramírez-Fráncel²; Gladys Reinoso-Flórez ${ }^{3}$
}

\begin{abstract}
'Licenciada en Educación Básica con Énfasis en Ciencias Naturales y Educación Ambiental, M.Sc., Ph.D.(c). Universidad del Tolima, Grupo de Investigación en Zoología, Ibagué - Tolima, Colombia; e-mail: Ivgarcia@ut.edu.co; Dhttps://orcid.org/0000-0001-7820-8977

²Licenciada en Educación Básica con Énfasis en Ciencias Naturales y Educación Ambiental, M.Sc., Ph.D.(c). Universidad del Tolima, Grupo de Investigación en Zoología, Ibagué - Tolima, Colombia; e-mail: laramirezfr@ut.edu.co; Dhttps://orcid.org/0000-0002-7144-3365
\end{abstract}

${ }^{3}$ Licenciada en Biología y Química, M.Sc. Universidad del Tolima, Departamento de Biología, Ibagué - Tolima, Colombia; e-mail: greinoso@ut.edu.co; (Dhttps://orcid.org/0000-0003-2002-1645

*autor de correspondencia: Ivgarcia@ut.edu.co

Cómo citar: García-Herrera, L.V.; Ramírez-Fráncel, L.A.; Reinoso-Flórez, G. 2019. Mamíferos del departamento del Tolima: distribución y estado de conservación. Rev. U.D.C.A Act. \& Div. Cient. 22(2):e1100. http://doi.org/10.31910/rudca.v22.n2.2019.1100

Artículo de acceso abierto publicado por Revista U.D.C.A Actualidad \& Divulgación Científica, bajo una licencia Creative Commons CC BY-NC 4.0

Recibido: Diciembre 3 de 2018

Aceptado: Julio 5 de 2019

Editado por: Ingeborg Zenner de Polanía

\section{RESUMEN}

El estudio mastozoológico del departamento del Tolima ha aumentado en los últimos años; sin embargo, esta información no ha sido compilada, ni actualizada, de manera que se identifiquen los errores de distribución y taxonómicos, en aras de identificar prioridades de investigación y de conservación. Con el fin de unificar la información de los mamíferos presentes en el departamento, se recopilaron y se validaron los registros museológicos de la Colección Zoológica de la Universidad del Tolima (CZUT-M), Museo Javeriano de Historia Natural Lorenzo Uribe (MPUJ), bases de datos de colecciones nacionales, internacionales e información bibliográfica. La lista de mamíferos consolidada confirma 126 especies, lo que equivale al $24,32 \%$ de las especies de mamíferos de Colombia, entre las cuales, se registra seis endémicas y siete en alguna categoría de amenaza. Esta diversidad, se agrupa en 30 familias, 88 géneros y 12 órdenes, en donde Chiroptera (71 spp.) y Rodentia (18 spp.) son los mejor representados. La zona de vida que evidenció la mayor riqueza de especies fue el Bosque Seco Tropical, con 56; no obstante, estas áreas han presentado un mayor esfuerzo investigativo en relación con otras, como las establecidas por encima de los $2.000 \mathrm{~m}$ s.n.m. Se resalta la necesidad de investigaciones en el departamento, para complementar los vacíos de información, así como la implementación de planes de manejo y de conservación, tanto de las especies como de las áreas.

Palabras clave: categoría de amenaza; colección biológica; endemismo; Chiroptera; Rodentia. 


\section{ABSTRACT}

The mastozoologica study of the department of Tolima has increased in recent years, however, this information has not been compiled, nor updated, so as to identify distribution and taxonomic errors in order to identify research and conservation priorities. In order to synthesize the information on mammals from this Department, here a compilation of information from voucher specimens from Colección Zoológica de la Universidad del Tolima (CZUT-M), Museo Javeriano de Historia Natural Lorenzo Uribe (MPUJ), national collections as well as records from international museum databases and the scientific literature were investigated. As product of our analyses, the list contains 126 species of mammals a number which is equivalent to $24.32 \%$ of the mammal species of Colombia; of these species, six are endemic to the country and seven are in any category of threat. This diversity is grouped into 30 families, 88 genera and 12 order, of these Chiroptera (71 spp.) and Rodentia (18 spp.) are the best represented. The zone of life that presented the greatest richness of species was the Tropical Dry Forest with 56, however, these areas have presented a greater investigative effort in relation to others, such as those established above 2000m.a.s.l. Our work reveals the need of new efforts to fill out the mammalian information gaps, as well as management and conservation plans for species and areas.

Keywords: biological collection; endemism; threatened category; Chiroptera; Rodentia.

\section{INTRODUCCIÓN}

Colombia ocupa la sexta posición mundial en términos de biodiversidad de mamíferos y el cuarto en el continente americano, con 518 especies (Ramírez-Chaves et al. 2016). Riqueza que se relaciona con la conexión al istmo de Panamá, epicentro de intercambio biótico entre el Norte y Suramérica y la compleja topografía dominada por los Andes, que determinó el levantamiento de las cordilleras Occidental, Central y, de esta última, la Oriental, separadas por las terrazas de los valles longitudinales de los ríos Cauca y Magdalena (Hernández-Camacho et al. 1992), que ofrecen una variedad bioclimática, hidrográfica y biológicas, únicas y contrastantes (Corredor-Prado \& Bejarano Bonilla, 2009).

Se ha identificado que la región andina presenta la mayor riqueza de especies, seguida por las regiones del Amazonas y el Pacífico (Solari et al. 2013); sin embargo, pese a la notable diversidad, esta región ha recibido una atención limitada, con vacíos de información en aspectos ecológicos y taxonómicos, para la mayoría de los grupos de mamíferos (Ramírez-Chaves et al. 2013). El departamento del Tolima, se ubica en la región Andina, entre las cordilleras Central y Oriental, cuenta con 47 municipios y una superficie de $23.582 \mathrm{~km}^{2}$, lo que representa el 2,1\% del territorio nacional; presenta 12 zonas de vida, distribuidas desde los 300 hasta los $5.000 \mathrm{~m}$ s.n.m., variedad geográfica que permite una diversidad de ecosistemas terrestres y acuáticos, así como tres Parques Naturales Nacionales (Los Nevados, Nevado del Huila y Las Hermosas), sistemas estratégicos en el contexto nacional, al albergar una alta diversidad de flora y fauna (Cortolima, 2007).

Los registros históricos de los mamíferos del departamento del Tolima son escasos; los primeros, corresponden a ocho individuos de Akodon affinis, colectados por L. E. Miller, en 1911, especímenes base para que, Allen (1912), describiera la especie, bajo el sinónimo de Akodon tolimae. Años más tarde, en 1944, el zoólogo y paleontólogo Ruben A. Stirton, recolectó dos especímenes de Myotis riparius, en el municipio del Carmen de Apicalá, cuando realizaba exploraciones de mamíferos fósiles en el país.

Posteriormente, la Universidad del Tolima, en su propósito de realizar una documentación detallada de la biodiversidad del departamento, encaminó los esfuerzos para evaluar la diversidad mastozoológica contenida en gran parte del territorio tolimense, iniciando con las investigaciones de Bejarano-Bonilla et al. (2007) y Galindo-Espinosa et al. (2010), como las primeras aproximaciones de la riqueza de murciélagos del departamento.

Los aportes más recientes, se realizaron entre el 2015 y 2018, con las investigaciones de García-Herrera et al. (2015), Ramírez-Fráncel et al. (2015), García-Herrera et al. (2018) y Ramírez-Fráncel et al. (2018), como resultado de estudios de caracterización biológica y exploraciones, que permitieron evaluar biomas estratégicos y la obtención de nuevos registros.

Con el fin de realizar una documentación detallada de la diversidad mastozoológica del departamento, se presenta la lista actualizada de los mamíferos del Tolima, con el objetivo de identificar y de llenar, tanto los vacíos de información en cubrimiento geográfico como corregir errores de distribución y taxonómicos.

\section{MATERIALES Y MÉTODOS}

La presente lista, se construyó con base en la revisión directa y confrontación taxonómica de todo el material biológico depositado en la Colección Zoológica de la Universidad del Tolima, sección mastozoología (CZUT-M, Ibagué), Museo Javeriano de Historia Natural Lorenzo Uribe (MPUJ, Bogotá) y a través de los registros históricos del Instituto de Ciencias Naturales de la Universidad Nacional (ICN, Colombia), American Museum of Natural History (AMNH, Estados Unidos), Museum of Vertebrate Zoology (MVZ, Estados Unidos) y el Royal Ontario Museum (ROM, Canadá), a través del portal Vernet (http://portal.vertnet.org). Además, se incluyó un proceso de búsqueda de información científica relacionada con el registro de mamíferos en el Tolima, disponible en Google Scholar, Google Books, ScienceDirect, Scopus, Scielo, Jstor (Bejarano-Bonilla et al. 2007; Galindo-Espinosa et al. 2009; Galindo-Espinosa et al. 2010; García-Herrera et al. 2015; GarcíaHerrera et al. 2018; Gutiérrez-Díaz et al. 2010; Mantilla-Meluk et al. 2009; Ramírez-Fráncel et al. 2015; Ramírez-Fráncel et al. 2018; Solari et al. 2013; Tarquino-Carbonell et al. 2016).

El presente listado taxonómico sigue la propuesta de Wilson \& Reeder (2005), con las modificaciones y las actualizaciones de 
Gardner (2008) y Díaz et al. (2016), en el orden Chiroptera; Patton et al. (2015), para Rodentia; Baker et al. (2016), en la clasificación de la familia Phyllostomidae y exclusión del género Dermanura; Velazco \& Patterson (2019), para inclusión de Sturnira giannae; Zurc et al. (2010), para el género Carollia; Rossi et al. (2010) y Voss et al. (2014), para el género Marmosa y la propuesta de Ruedas et al. (2017), para las especies de Sylvilagus.

Para cada espécimen, se tomaron medidas craneales y externas, usando un calibrador digital de precisión $0,1 \mathrm{~mm}$ : longitud mayor del cráneo (LMC): desde el punto más posterior del occipital hasta el punto más anterior del premaxilar (excluyendo los incisivos); longitud condilobasal (LCB): desde el punto más posterior en los cóndilos occipitales hasta el punto anterior de los incisivos superiores y anchura cigomática (ZB): tomada como la amplitud, a través de los arcos cigomáticos. Las medidas externas, se tomaron de las etiquetas de los especímenes: longitud total (LT): distancia desde la punta del hocico a la última vértebra caudal, para marsupiales, roedores y carnívoros y, hasta el borde del uropatagio, para murciélagos; longitud de la oreja (LO): desde la muesca hasta la punta carnosa del pabellón auricular; longitud del pie trasero (LP): desde el margen proximal del calcáneo hasta la punta de la garra del dedo más largo y longitud de la cola (LC): medida desde el punto de flexión del sacro hasta la punta de la última vértebra caudal.

A través de la revisión del material biológico de la CZU-UT y MPUJ, así como la consulta de las bases de datos de colecciones extranjeras, se recopiló información sobre especies de mamíferos (nativos), procedente de 37 municipios del departamento (Figura 1).

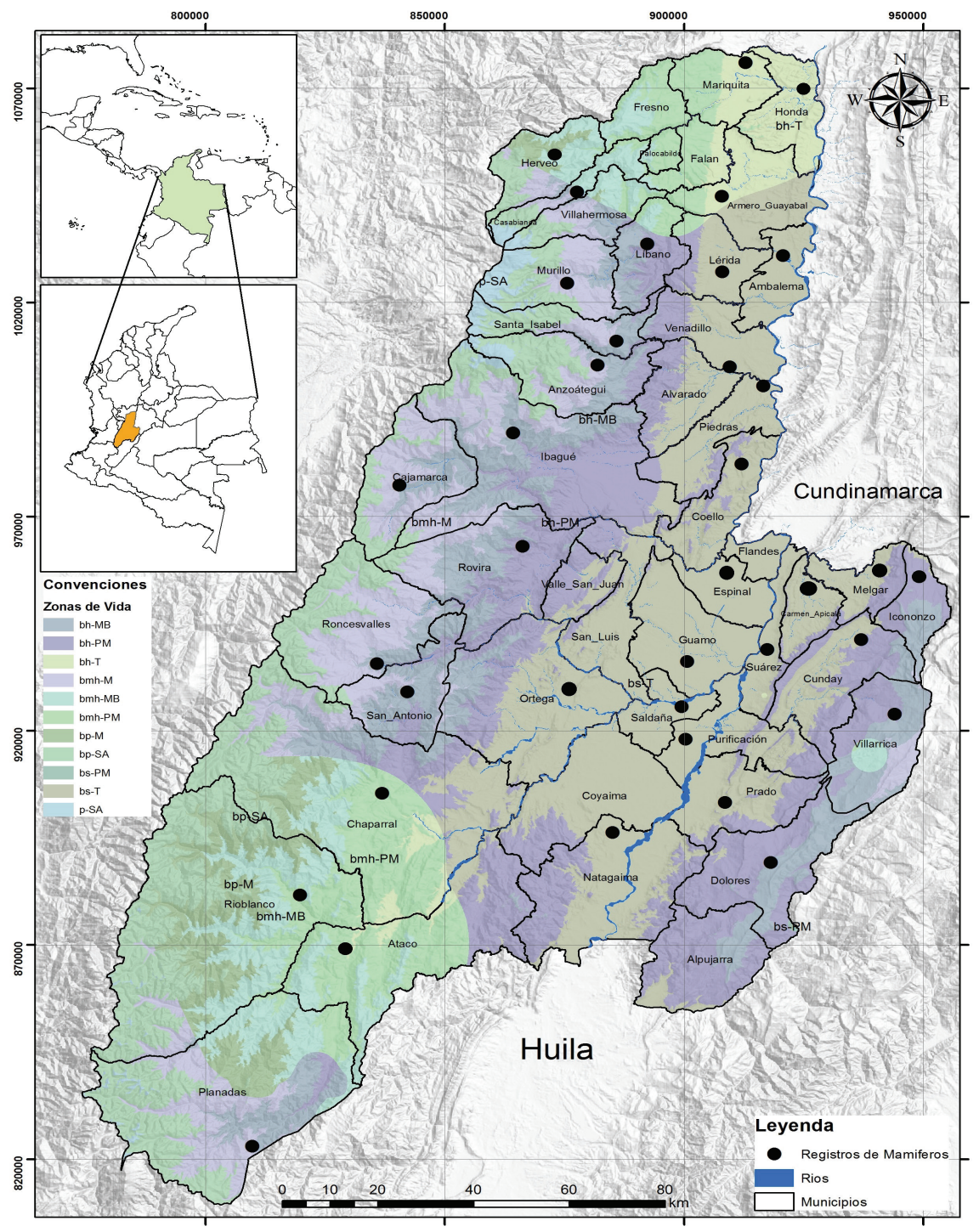

Figura 1. Localidades del material revisado, preservado en la Colección Zoológica de la Universidad del Tolima, sección mastozoología (CZUT-M), Colombia. bsT: Bosque seco tropical; bhT: Bosque húmedo tropical; bsPM: Bosque seco premontano; bhPM: Bosque húmedo premontano; bmhPM: Bosque muy húmedo premontano; bhMB: Bosque húmedo montano bajo; bmhMB: Bosque muy húmedo montano bajo; bpM: Bosque pluvial montano; bpSA: Bosque pluvial subalpino; pSA: Páramo subalpino. 


\section{RESULTADOS Y DISCUSIÓN}

La lista de mamíferos del Tolima está compuesta por 126 especies, agrupadas en 88 géneros, 30 familias y 12 órdenes (Apéndice 1). Riqueza similar a la registrada por Castaño et al. (2017), en el departamento de Risaralda (134 especies, 88 géneros, 32 familias y 12 órdenes), abarcando el 24,32\% de los mamíferos de Colombia, de acuerdo con lo reportado por Ramírez-Chaves et al. (2016). Los órdenes mejor representados fueron Chiroptera, con 71 especies (56,34\%), seguido por Rodentia, con 18 spp. $(14,28 \%)$ y Carnivora, con 15 spp. (11,90\%). Por otra parte, los órdenes con menor porcentaje fueron: Didelphimorphia, con $8 \mathrm{spp}$. (6,34\%); Primates, con 4 spp. (3,17\%); Artiodactyla, con 3 spp. (2,38\%) y Pilosa, con 2 spp. (1,58\%), mientras los órdenes Paucituberculata, Cingulata, Soricomorpha, Perissodactyla y Lagomorpha registraron una sola especie $(0,79 \%)$, respectivamente. La composición de los mamíferos del Tolima sigue el patrón de riqueza, registrado a nivel mundial, en donde Chiroptera y Rodentia son los órdenes con mayor número de especies (Burgin et al. 2018).

Un total de 30 familias tienen distribución en el departamento del Tolima, de las cuales, Phyllostomidae (Chiroptera) y Cricetidae (Rodentia), se muestran como las mejor representadas, con 47 y 11 especies, respectivamente, seguido de Vespertilionidae (Chiroptera), con 10 especies y Didelphidae, con ocho. Es de aclarar que el número de especies de murciélagos del Tolima, consignados por GalindoEspinosa et al. (2010), es superior al registrado en este trabajo (72 ssp.), debido a identificaciones erróneas, por lo que fueron excluidos del presente listado (Lonchophylla mordax, Sturnira magna, Sturnira mordax, Platyrrbinus aurarius) y se reasignaron taxonómicamente a otras especies (Apéndice 1).

El mayor número de taxones, se reporta en la zona de vida bs-T (por debajo de los $1.000 \mathrm{~m}$ s.n.m.; 57 spp.), debido a que las zonas altas (por encima de los $2.000 \mathrm{~m}$ s.n.m.), han sido escasamente evaluadas, dado los problemas de movilidad y de orden público que enfrentó el país. Estos vacíos de información dificultan la definición de áreas con interés prioritario de conservación y las futuras medidas tendientes de protección a la mastofauna. En el Tolima habitan, por lo menos, seis especies endémicas de Colombia; tres roedores: Akodon affinis (Allen, 1912) Nephelomys childi (Thomas, 1895) y Thomasomys cinereiventer (J.A. Allen, 1912); dos primates: Aotus griseimembra Elliot, 1912 y Saguinus leucopus (Günther, 1877) y la musaraña Cryptotis medellinius (Thomas, 1921). Asimismo, se reportan siete especies en algún estado de amenaza, a nivel mundial (UICN, 2018); en peligro (EN), la danta de páramo Tapirus pinchaque (Roulin, 1829) y el titi gris $S$. leucopus y cinco especies en categoría de vulnerable (VU), el tigrillo Leopardus tigrinus (Schreber, 1775), el oso de anteojos Tremarctos ornatus (F.G. Cuvier, 1825), los monos de la noche Aotus griseimembra, A. lemurinus (I. Geoffroy, 1843) y el venado conejo Pudu mephistophiles (de Winton, 1896). Adicionalmente, la nutria de río Lontra longicaudis (Olfers, 1818) está catalogada como vulnerable para el país, de acuerdo con la Resolución 1912 de 2017, expedida por el Ministerio de Ambiente y Desarrollo Sostenible.
Además de las especies que están catalogadas en algún grado de amenaza es importante considerar que la mastofauna en el Tolima, se encuentra bajo una fuerte presión, a causa de la cacería, la reducción, la destrucción de los bosques y el empleo en rituales culturales; Potos flavus (Schreber, 1774) es sacrificada por las comunidades indígenas del suroeste del departamento, por considerarse portadora de espíritus del mal, mientras que las especies Didelphis marsupialis Linnaeus, 1758, Coendou pruinosus Thomas, 1905 Cuniculus paca (Linnaeus, 1766), Cuniculus taczanowskii (Stolzmann, 1865) y Dasyprocta punctata Gray, 1842, suelen ser empleadas como fuente proteica, y, a su vez, Tamandua mexicana (Saussure, 1860) y Procyon cancrivorus (G. Cuvier, 1798), se utilizan para extracción de piel/cuero.

A partir de la revisión realizada, se adicionan seis nuevos registros, que extienden la distribución geográfica de algunas especies en Colombia:

Orden Didelphimorphia Gill, 1872, Family Didelphidae Gray, 1821. Marmosa isthmica Goldman, 1912.

Material revisado: 2 machos adultos (CZUT-M 0644, 1185) у 2 hembras adultas (CZUT-M 1184, 1186), colectados en los municipios de Ibagué (1.795m s.n.m.) y Ambalema (250m s.n.m.).

Identificación: Se consultaron las descripciones y las medidas de Marmosa isthmica, proporcionadas por Goldman (1912) y Voss et al. (2014). Los especímenes de los municipios de Ambalema e Ibagué exhiben las características de diagnóstico de la especie: pelaje dorsal marrón; rostro claro con área orbital de color negro; coloración de la base de los dedos de las patas anteriores marrón y dedos blanquecinos; pelaje ventral de base gris visible, pero restringido a los lados del abdomen, pelaje central claro, extendiéndose como una línea mediana continua, desde la barbilla hasta el ano; tamaño corporal mediano (LT:230-238, LO:16-34, LP:24-25, LC:215-218 $\mathrm{mm}$ ); rostro estrecho, surco suborbital fuertemente desarrollado, falta de fenestra palatina y bullas auditivas relativamente pequeñas, cráneo grande (Figura 2a; LMC: 44,5-44,8; LCB: 40-43; ZB: 23,6$23,5 \mathrm{~mm})$.

La distribución conocida previamente para la especie comprende a los departamentos de Antioquia, Caldas, Chocó, Córdoba, Cundinamarca, Nariño, Sucre, Valle del Cauca en la franja de los 0 hasta los $1.120 \mathrm{~m}$ s.n.m. (Solari et al. 2013) y Risaralda, de los 300 hasta los $1.300 \mathrm{~m}$ s.n.m. (Castaño et al. 2017). Este reporte, en fragmentos de Bosque Seco Tropical en el Valle alto del río Magdalena y en el Bosque húmedo montano bajo, amplía su distribución en la región andina.

\section{Marmosops parvidens (Tate, 1931)}

Material revisado: Cuatro machos adultos (CZUT-M 0641, 0858, 0872, 0891), colectados en Ibagué (1.795m s.n.m.) y tres machos (CZUT-M 1194, 1195, 1196), procedentes del municipio de Murillo (2.600m s.n.m.).

Identificación: Se consultaron las descripciones y las medidas de Marmosops parvidens, proporcionadas por Gardner (2008) y DíazNieto et al. (2016). Los especímenes evaluados exhibieron los caracteres diagnósticos: coloración dorsal marrón rojizo y pelaje ventral crema, desde la barbilla hasta el ano (incluidas las superficies internas de las extremidades anteriores y posteriores), con bordes 


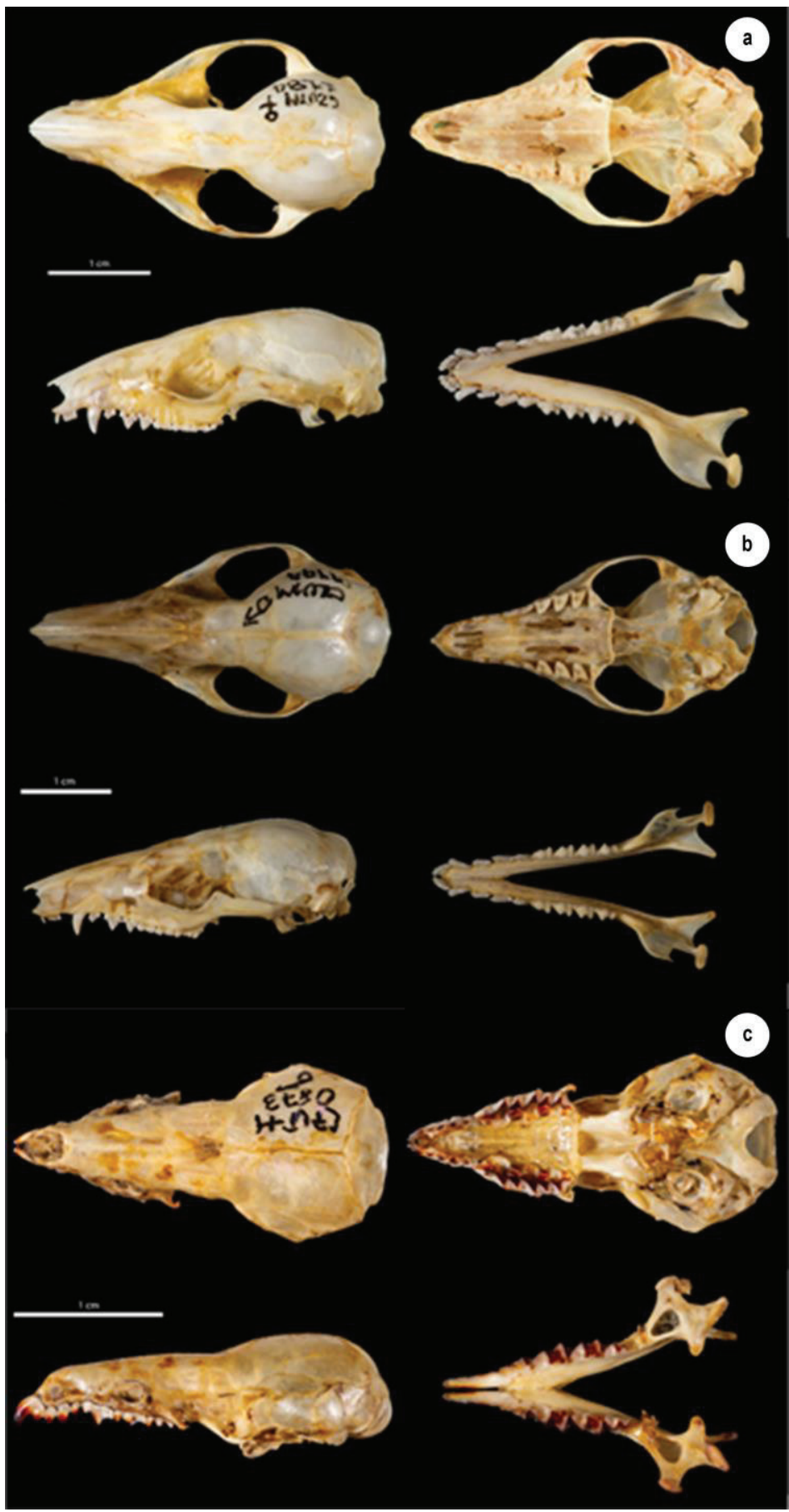

Figura 2. Vistas dorsal, ventral y lateral del cráneo y vista dorsal de la mandíbula. A) Marmosa isthmica (CZUT-M 1184); b) Marmosops parvidens (CZUT-M 1194); c) Cryptotis medellinius (CZUT-M 0874) Barra de escala $=1 \mathrm{~cm}$.

de color gris en la base; huesos nasales largos (que se extienden por detrás de los lagrimales); el foramen lagrimal, generalmente, se oculta de la vista lateral dentro del margen orbital anterior; proceso cigomático del escamoso ampliamente superpuesto dorsalmente por el yugal; palatina fenestra ausente, cráneo mediano (Figura 2b; LMC:27,3-29,1; LCB: 24,9-26; ZB: 13,8-15mm); tamaño pequeño (LT: 143-161; LO: 19-21; LP: 15,5-16,4; LC: 115-117mm).
Marmosops parvidens ha sido reportada en los departamentos de Antioquia, Boyacá, Caldas, Cauca, Huila y Vaupés, a 1.400m s.n.m. (Solari et al. 2013). Por lo tanto, los registros en la zona de vida Bosque húmedo pre-montano y Bosque húmedo montano bajo del Tolima complementan la distribución geográfica conocida para la especie.

Soricomorpha Gregory, 1910; Soricidae Fischer von Waldheim, 1817 Cryptotis medellinius Thomas, 1921 
Material revisado: 1 macho adulto (CZUT-M 0873), 1 hembra adulta (CZUT-M 0874), colectados en la Reserva Forestal Bella Vista, kilómetro 96, Ibagué (2.468m s.n.m.).

Identificación: Se consultaron las descripciones y las medidas de Cryptotis medellinius, proporcionadas por Thomas (1921) y Gardner (2008). Nuestros especímenes exhiben las características, diagnóstico de la especie: tamaño del cuerpo pequeño y cola corta (LT 87-94mm; LC: 26-27; LP:13-16; y LO: 5,2-7,9 mm); la coloración general del pelaje es grisácea y casi uniforme con el vientre; pelos del dorso de $6,2 \mathrm{~mm}$ de largo; dígitos, tanto anteriores como posteriores blancuzco; foramen en el borde posterior del proceso timpánico del petromastoideo de tamaño grande y el borde anterior del proceso coronoideo unido a la rama horizontal de la mandíbula (Figura 2c; LMC:20,2-21,6; LCB:19,5-20; AZ:1,8-1,9; LT: 73-98; LC: 23-41; LP:13-16 y LO: 6,2-7,9 mm).

La especie se ha reportado en el norte de la cordillera central y occidental, en los departamentos de Antioquia, Caldas y Risaralda, dentro de un rango de elevación de 1.800 a 3.800m s.n.m. (NogueraUrbano et al. 2019), en los hábitats: bosque húmedo premontano, bosque bajo montano, bosque montano y selva baja montano (Cuartas-Calle y Muñoz Arango, 2003). Este registro en Ibagué extiende la distribución geográfica, adicionando el ecosistema de bosque muy húmedo pre-montano.

Chiroptera Blumenbach, 1779; Phyllostomidae Gray, 1825

Micronycteris microtis Miller, 1898

Material revisado: 1 hembra adulta (CZUT-M 0717), recolectado en el municipio de Venadillo (700m s.n.m.) y 2 machos adultos (CZUT-M 1085, 1725), de los municipios de Roncesvalles $(1.500 \mathrm{~m}$ s.n.m.). y Ambalema (251m s.n.m.), respectivamente.

Identificación: Se consultaron las descripciones y las mediciones de Micronycteris microtis, proporcionadas por Miller (1898) y Simmons y Voss (1998). Los especímenes evaluados exhiben coloración dorsal marrón grisáceo, pelos largos $(7-8 \mathrm{~mm})$, bicoloreados con $1 / 4$ de las base blanca e incisivos bífidos, cráneo pequeño (Figura 3a; LMC:17,56-18,06; LCB:16,50-16-89; ZB:8,42-8,99mm), orejas redondeadas, unidas mediante una banda de piel y área adyacente desnuda (LT:65-68,6; LA:33,25-33,44; LC:13,88-15,5; LP:9,95-10,5; LO: $15,5-18,53 \mathrm{~mm})$.

La distribución conocida para la especie, de acuerdo con lo reportado por Solari et al. (2013), se establece en la región Andina (Cundinamarca) y Caribe (Magdalena). Mediante el examen de caracteres morfológicos, se establece que los especímenes $M$. microtis registrados, se constituye en los primeros datos para la cordillera central del país.

Platyrrbinus infuscus (Peters, 1880)

Material revisado: 1 macho adulto (CZUT-M 0586) del municipio de Natagaima (333m s.n.m.) y 1 hembra adulta (CZUT-M 0198) de Ibagué (1,777 m s.n.m.).

Identificación: Se consultaron las descripciones y medidas de Platyrrbinus infuscus, proporcionadas por Velazco \& Gardner (2009). Nuestros ejemplares exhiben las características diagnósticas de la especie: tamaño grande (FA> 57 mm); pelaje dorsal marrón oscuro; líneas faciales y dorsales discretas; las líneas superiores nacen detrás de la nariz, pasan sobre los ojos, terminando en la base superior de las orejas y las líneas inferiores nacen en la base del labio superior, pasando debajo de los ojos, terminando en la base inferior de las orejas, pelo corto y escaso en la superficie superior de los pies; cráneo robusto, hocico ancho y corto; incisivos superiores centrales convergentes y anchos, presencia de tres molares superiores, $\mathrm{p} 4$ con talón postero-labial reducido, cúspide posterolabial presente en p 5 y parastilo presente en M1. Los individuos analizados presentaron las siguientes medidas craneales y corporales (Figura 3b; LMC:31,46-32; LCB: 25,57- 26,1; ZB:18,27-18,56; LT: 66,4- 68,8; LA: 57,45-58,10; LP: 10,1-15,5; LO:12,0-13,7mm).

En Colombia esta especie ha sido reportada en la Amazonia, la Serranía de La Macarena, cordillera Oriental y en los valles interandinos, entre los 0 a los $1.300 \mathrm{~m}$ s.n.m. Este registro extiende la distribución geográfica conocida para la especie en el país, adicionando el ecosistema Bosque seco pre-montano.

Carnivora Bowdich, 1821; Procyonidae Gray, 1825

Nasuella olivacea (Gray, 1865)

Material revisado: 1 machos adulto (CZUT-M 0918), en Ibagué (3.056m s.n.m.) y 1 hembra adulta (CZUT-M 0537), recolectada en el municipio de Cajamarca (2.646m s.n.m.).

Identificación: Se consultaron las descripciones y las mediciones propuestos por Decker \& Wozencraft (1991). Los ejemplares presentan hocico triangular, coloración del dorso tricolor (gris cenizo, negro y ocre), frente y mejillas con pelos bicolor (base negra y puntas blancas), vientre bicolor (base gris y puntas blancas), ausencia del proceso supraorbital y cresta sagital (Figura 3c; LMC:72,71-74,09; LCB:59,94-59,18; AZ:28,81-31,52; LT: 220-360; LC: 110-128; LP: 46,45-55; LO: 26-26,85mm).

Se ha propuesto que la especie tiene distribución en los departamentos de Antioquia, Boyacá, Cauca, Cesar, Cundinamarca, Meta, Nariño y Santander, entre los 1.700 a los $4.100 \mathrm{~m}$ s.n.m. (Solari et al. 2013). Los especímenes de Cajamarca e Ibagué, se constituyen en el primer registro confirmado para el Tolima, en el ecosistema Bosque húmedo montano bajo.

Se recomienda una revisión profunda del ejemplar Aotus griseimembra (CZUT-M 1737), en aras de aclarar los debates sobre su identidad taxonómica y distribución. Asimismo, es necesario ampliar la evaluación de la mastofauna a los municipios faltantes; estos vacíos de información dificultan definir con respaldo científico las áreas de alta diversidad, insumo indispensable para aumentar el conocimiento de este grupo, el diseño de planes y programas para su manejo y conservación.

Agradecimientos: Los autores agradecen a Sergio Solari por su apoyo y valiosa colaboración en la corroboración de los especímenes del orden Chiroptera y Rodentia, depositados en la Colección Zoológica de la Universidad del Tolima sección mastozoología (CZUT-M). A las instituciones que han financiado la evaluación de la mastofauna del Tolima, a la Corporación Autónoma Regional del Tolima, a la Universidad del Tolima, al Fondo de Investigaciones y 

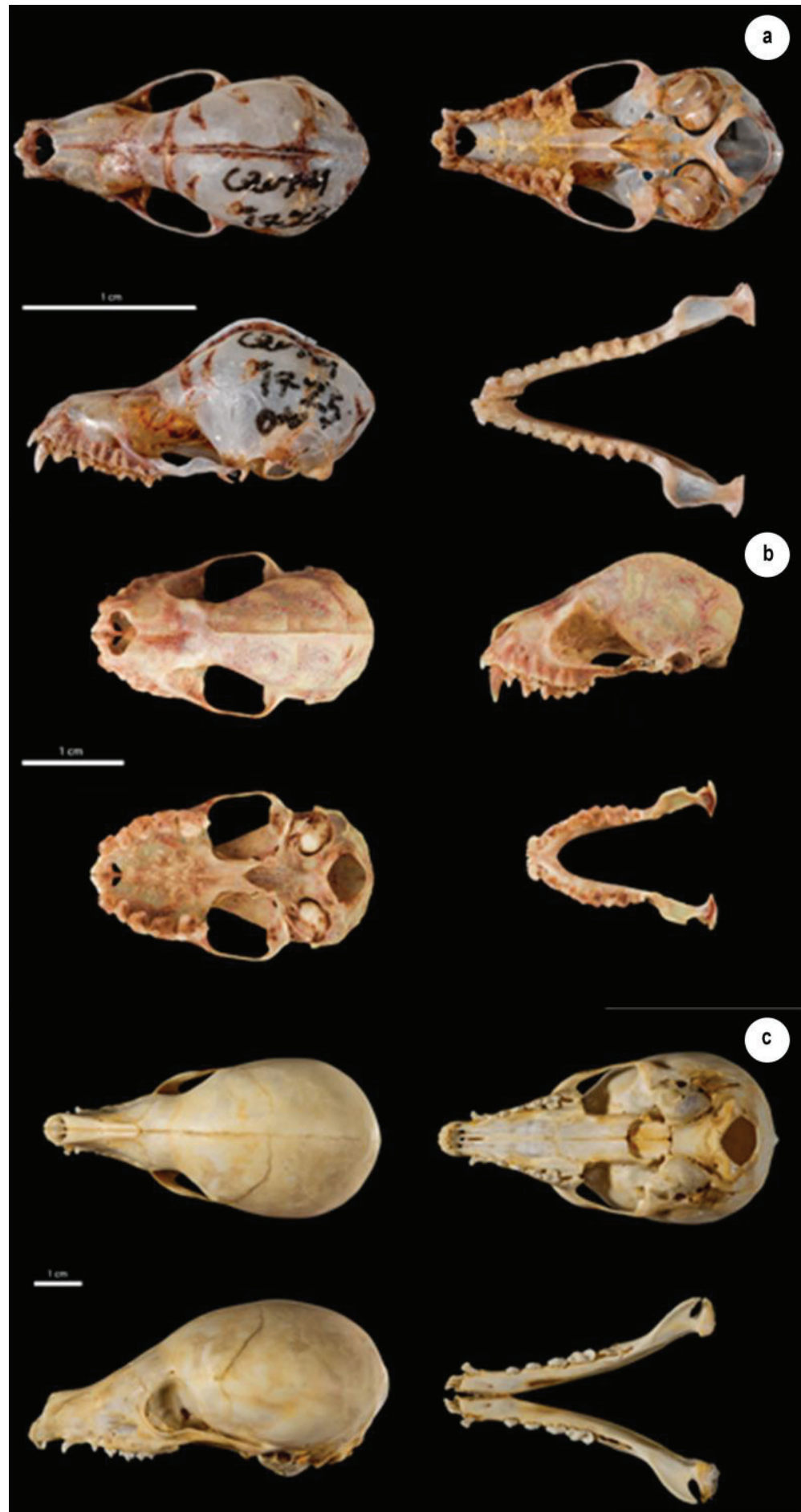

Figura 3. Vistas dorsal, ventral y lateral del cráneo y vista dorsal de la mandíbula. A) Micronycteris microtis (CZUT-M 1725); b) Platyrrbinus infuscus (CZUT-M 0586); c) Nasuella olivacea (CZUT-M 0537). Barra de escala $=1 \mathrm{~cm}$.

Desarrollo Científico y a los investigadores, quienes hicieron parte de la evaluación de la mastofauna del Tolima. A la Colección Zoológica de la Universidad del Tolima y Museo de Historia Natural Lorenzo Uribe Uribe S. J. de la Pontificia Universidad Javeriana, así como a Jairo Pérez, por la asistencia en el trabajo museológico. LVGH y LARF agradecen a la convocatoria 755 de 2016 de Colciencias para estudios de doctorado nacionales, tiempo, en el cual, se elaboró este artículo. 
Conflicto de intereses: El manuscrito fue preparado y revisado con la participación de todos los autores, quienes declaramos que no existe conflicto de intereses que ponga en riesgo la validez de los resultados presentados.

\section{REFERENCIAS}

1. ALLEN, J.A. 1912. Mammals from western Colombia. Bulletin of the American Museum of Natural History (1):71-79.

2. BAKER, R.J.; SOLARI, S.; CIRRANELLO, A.; SIMMONS N.B. 2016. Higher level classification of phyllostomid bats with a summary of DNA synapomorphies. Acta Chiropterologica 18:1-38. https://doi. org/10.3161/15081109ACC2016.18.1.001

3. BEJARANO-BONILLA, D.A.; YATE-RIVAS, A.; BernalBautista, M.H. 2007. Diversidad y distribución de la fauna quiróptera en un transecto altitudinal en el departamento del Tolima, Colombia. Caldasia 29(2):297-308. https:/ / doi. org/10.13140/RG.2.1.1336.7520

4. BURGIN, C.J.; COLELLA, J.P.; KAHN, P.L.; UPHAM, N.S. 2018. How many species of mammals are there? J. Mammalogy. 99(1):1-14. https://doi.org/10.1093/ jmammal/gyx147

5. CASTAÑO, J.H.; TORRES, D.; ROJAS-DÍAZ, V.; SAAVEDRA-RODRÍGUEZ, C.; PÉREZ-TORRES, J. 2017. Mamíferos de Risaralda, Colombia. Biota Colombiana 18(2):239-253. https://doi.org/10.21068/c2017.v18n02a16

6. CORREDOR-PRADO, J.P.; BEJARANO BONILLA, D.A. 2009. Pequeños mamíferos no voladores de la reserva natural Ibanasca (Tolima, Colombia). Tumbaga. 1(4):121134.

7. CORPORACIÓN AUTÓNOMA REGIONAL DEL TOLIMA, CORTOLIMA. 2007.- Plan de Acción Trienal. 2007-2009.

8. CORPORACIÓN AUTÓNOMA REGIONAL DEL TOlimA, CORTOLIMA. 2016.- Plan de Manejo Ambiental (PMA) Paramo del Meridiano.

9. CUARTAS-CALLE, C.A.; MUÑOZ-ARANGO, J. 2003. Marsupiales, cenoléstidos e insectívoros de Colombia. Editorial Universidad de Antioquia. Medellín, Colombia.

10. DECKER, D.M.; WOZENCRAFT, W.C. 1991. Phylogenetic analysis of recent procyonid genera. J. Mammalogy. 72(1):42-55. https://doi.org/10.2307/1381979

11. DÍAZ, M.M.; SOLARI, S.; AGUIRRE, L.F.; AGUIAR, L.M.S.; BARQUEZ, R.M. 2016. Clave de Identificación de Los Murciélagos del Cono Sur de Sudamérica. Publicación especial $\mathrm{N}^{\circ}$ 2, PCMA. Programa de Conservación de los Murciélagos de Argentina. Argentina. p.32-146.

12. DÍAZ-NIETO, J.F.; JANSA, S.A.; VOSS, R.S. 2016. DNA sequencing reveals unexpected recent diversity and an ancient dichotomy in the American marsupialgenus Marmosops (Didelphidae: Thylamyini). Zoological J. Linnean Society. 176:914-940. https://doi.org/10.1111/ zoj. 12343

13. GALINDO-ESPINOSA, E.; GUTIÉRREZ-DÍAZ, K.; YARAORTIZ, D.; REINOSO-FLÓREZ, G.; VEJARANODELGADO, M.; GARCÍA-MELO, J. 2009. Plan de Manejo Regional para la Conservación del titi gris Saguinus leucopus en el departamento del Tolima. Ibagué, Colombia. p.13-98.

14. GALINDO-ESPINOSA, E.; GUTIÉRREZ-DIAZ, K.; REINOSO-FLÓREZ, G. 2010. Lista de los quirópteros del departamento del Tolima, Colombia. Biota Colombiana. 11(1):107-116.

15. GARCÍA-HERRERA, L.; RAMÍREZ-FRÁNCEL，L; REINOSO-FLÓREZ, G. 2015. Mamíferos en relictos de bosque seco tropical del Tolima, Colombia. Mastozoología Neotropical. 22(1):11-21.

16. GARCÍA-HERRERA，L.; RAMÍREZ-FRÁNCEL，L.; REINOSO FLÓREZ, G. 2018. Potential distribution of Sphaeronycteris toxophyllum in Colombia and new record. Therya. 9(3):255-260. https://doi.org/10.12933/ therya-18-610

17. GARDNER, A.L. (ed.). 2008. Mammals of South America. Volume 1: Marsupials, Xenarthrans, Shrews, and Bats. University of Chicago Press, Chicago, Illinois, and London, United Kingdom. p.1-484.

18. GOLDMAN, E.A. 1912. Descriptions of twelve new species and subspecies of mammals from Panama. Smithsonian Miscellaneous Collections. 56(36):1-11.

19. GUTIÉRREZ-DÍAZ, K.; GALINDO-ESPINOSA, E.; YARAORTIZ, D.; REINOSO- FLÓREZ, G.; VEJARANODELGADO, M.; GARCÍA-MELO, J. 2009. Plan de Manejo Regional para la Conservación de la danta de páramo (Tapirus pinchaque) en el departamento del Tolima. Ibagué, Colombia. p.4-119.

20. GUTIÉRREZ-DÍAZ，K.; GALINDO-ESPINOSA，E.; REINOSO- FLÓREZ, G. 2010. Nuevos registros de quirópteros para el departamento del Tolima, Colombia. Tumbaga. 5:39-40.

21. HERNÁNDEZ-CAMACHO, J.; WALSCHBURGER, T; ORTÍZ, R.; HURTADO, A. 1992. Origen y distribución 
de la biota suramericana y colombiana. En: Halffter, G. (Comp.). La diversidad biológica de Iberoamérica I. Acta Zoológica Mexicana. Volumen especial. CYTED-D. Programa Iberoamericano de Ciencia y Tecnología para el Desarrollo. México. p.55-104.

22. NOGUERA-URBANO, E.A.; COLMENARES-PINZÓN, J.E.; VILLOTA, J.; RODRÍGUEZ-BOLAÑOS, A.; RAMÍREZ-CHAVES, H.E. 2019. The shrews (Cryptotis) of Colombia: What do we know about them? Therya 10 (2):131-147. https://doi.org/10.12933/therya-19-760

23. MANTILLA-MELUK, H.; JIMÉNEZ-ORTEGA, A.M.; BAKER, R.J. 2009. Phyllostomid Bats of Colombia: Annotated Checklist, Distribution and Biogeography. Special Publications Museum of Texas Tech University. 56:1-37.

24. MiLLER, G.S. 1898. Descriptions of five new Phyllostome bats Proc. Academy of Natural Sciences of Philadelphia. 50:326-337.

25. MINISTERIO DEL AMBIENTE Y DESARROLLO SOSTENIBLE. 2017. Resolución 1912 de 2017.

26. PATTON, J.; PARDIÑAS, U.; ELÍA, G. 2015. Mammals of South America, Volume 2 - Rodents. The University of Chicago Press. Chicago. p.1-1048. https://doi. org/10.7208/chicago/9780226169606.001.0001

27. RAMÍREZ-CHAVES, H.E.; NOGUERA-URBANO, E.A.; RODRÍGUEZ-POSADA, M.E. 2013. Mamíferos (Mammalia) del departamento de Putumayo, Colombia. Rev. Academia Colombiana de Ciencias Exactas, Físicas y Naturales. 37:263-286. https://doi.org/10.18257/ raccefyn.9

28. RAMÍREZ-CHAVES, H.E.; SUÁREZ-CASTRO, A.E.; GONZÁLEZ-MAYA, J.F. 2016. Cambios recientes a la lista de los mamíferos de Colombia. Mammalogy Notes/ Notas Mastozoológicas. 3:1-9.

29. RAMÍREZ-FRÁNCEL, L.; GARCÍA-HERRERA, L.; REINOSO-FLÓREZ, G. 2015. Nuevo registro del murciélago pálido Phylloderma stenops (Phyllostomidae); en el valle alto del río Magdalena, Colombia. Mastozoología Neotropical. 22(1):97-102.

30. RAMÍREZ-FRÁNCEL, L.; GARCÍA-HERRERA, L.; REINOSO-FLÓREZ, G. 2018. First record of Platyrrbinus albericoi Velazco, 2005 (Chiroptera, Phyllostomidae) in the eastern slope of the Central Andes of Colombia. Check List. 14:1161-1167. https://doi.org/10.15560/14.6.1161
31. ROSSI, R.; VOSS, R.; LUNDE, D. 2010. A revision of the didelphid marsupial genus Marmosa. Part 1, The species in Tate's 'mexicana' and 'mitis' sections and other closely related forms. Bulletin of the American of Natural History. 334:1-83. https://doi.org/10.1206/334.1

32. RUEDAS, L.A.; FRENCH, J.H.; SILVA, S.M.; PLAT'T, I.L.; NELSON, R.; SALAZAR-BRAVO, J.; THOMPSON, C.V. 2017. A prolegomenon to the systematics of South American cottontail rabbits (Mammalia, Lagomorpha, Leporidae: Sylvilagus): Designation of a Neotype for $S$. brasiliensis (Linnaeus, 1758), and restoration of S. andinus (Thomas, 1897) and S. tapetillus Thomas, 1913. Miscellaneous Publications. Museum of Zoology, University of Michigan. 205:1-67. https://doi.org/10.1093/jmammal/gyx133

33. SIMMONS, N.B.; VOSS, R.S. 1998. The mammals of Paracou, French Guiana: a Neotropical lowland rainforest fauna. Part 1. Bats Bulletin of the American Museum of Natural History. 237:1-219.

34. SOLARI, S.; MUÑOZ-SABA, Y.; RODRÍGUEZ-MAHECHA, J.V.; DEFLER, T.R.; RAMÍREZ-CHAVES, H.E.; TRUJILLO, F. 2013. Riqueza, endemismo y conservación de los mamíferos de Colombia. Mastozoología Neotropical. 20(2):301-365.

35. TARQUINO-CARBONELL, A.; GUTIÉRREZ-DÍAZ, K.; GALINDO-ESPINOSA, E.; REINOSO-FLÓREZ, G.; SOLARI, S.; GUERRERO, R. 2016. Ectoparasites associated with bats in northeastern Tolima, Colombia. Mastozoología Neotropical. 22(2):349-358.

36. THOMAS, O. 1921. New Cryptotis, Thomasomys, and Oryzomys from Colombia. The Annals and Magazine of Natural History. 9:354-57.

37. UNIÓN INTERNACIONAL PARA LA CONSERVACIÓN DE LA NATURALEZA, UICN. 2018. http://dx.doi. org/10.2305/IUCN.UK.2016-1.RLTS.T21473A45173922. en

38. VELAZCO, P.M. 2005. Morphological Phylogeny of the bat Genus Platyrrhinus Saussure, 1860 (Chiroptera: Phyllostomidae) with the description of four new species. Fieldiana Zoology, New Series. 105:1-53. https://doi. org/10.5962/bhl.title.2689.

39. VELAZCO, P.M.; GARDNER, A.L. 2009. A new species of Platyrrhinus (Chiroptera: Phyllostomidae) from western Colombia and Ecuador, with emended diagnoses of $P$. aquilus, $P$. dorsalis, and $P$. umbratus. Proc. Biological Society of Washington. 122(3):249-281. https://doi. org/10.2988/08-40.1 
40. VELAZCO, P.M.; PATTERSON, B.D. 2019. Small mammals of the Mayo River Basin in northern Peru, with the escription of a new species of Sturnira (Chiroptera, Phyllostomidae). Bulletin of the American Museum of Natural History. 429:1-7. http://digitallibrary.amnh.org/handle/2246/6933

41. VOSS, R.S.; GUTIÉRREZ, E.E.; SOLARI, S.; ROSSI, R.V.; JANSA, S.A. 2014. Phylogenetic relationships of mouse Opossums (Didelphidae, Marmosa) with a revised subgeneric classification and notes on sympatric diversity. American Museum Novitates. 3817:1-27. https://doi. org/10.1206/3817.1
42. WILSON, D.E.; REEDER, D.M. 2005. Mammal species of the World: a taxonomic and geographic reference. Johns Hopkins University Press. Baltimore. 2142p.

43. YARA-ORTIZ, D.; GALINDO-ESPINOSA, E.; GUTIÉRREZ-DÍAZ，K.; REINOSO-FLÓREZ，G.; BEJARANO-DELGADO, M.; GARCÍA-MELO, J. 2009. Plan de Manejo Regional para la Conservación del puma o león de montaña (Puma concolor) en el departamento del Tolima. Ibagué, Colombia. p.4-119.

44. ZURC, D.; VELAZCO, P. 2010. Análisis morfológico y morfométrico de Carollia colombiana Cuartas et al. 2001 y $C$. monohernandezi Muñoz et al. 2004 (Phyllostomidae: Carolliinae) en Colombia. Chiroptera Neotropical. 16:549-567. 